\section{Monocytes feel the pressure}

myeloid cells,
through
PIEZO1, can
sense pressure
alterations
in their
environment
and integrate
this with a pro-
inflammatory
response

Immune cells are endowed with the ability to simultaneously sense numerous signals in their microenvironment and tailor their response accordingly. Besides sensing signals derived from pathogens and physiological changes in temperature, $\mathrm{pH}$, oxygen and nutrient levels, a study in Nature describes a new pathway in which innate immune cells sense physical forces as well. The authors show, in mice, that this mechanosensation by monocytes in the lungs is crucial for protection from pulmonary bacterial infection.

Cells in the lungs are exposed to repeated mechanical forces and pressures when tissues are compressed during breathing, but little is known about how this affects the behaviour of infiltrating immune cells. The first clue that myeloid cells might respond to such physical forces came from the observation that bone marrow-derived macrophages (BMDMs) express high levels of the mechanically activated calcium channel PIEZO1.

The authors used a pressure chamber to mimic the cyclical hydrostatic pressure (CHP) that immune cells experience in the lungs. After a $6 \mathrm{~h}$ incubation of BMDMs in the pressure chamber, RNA sequencing analysis revealed a robust

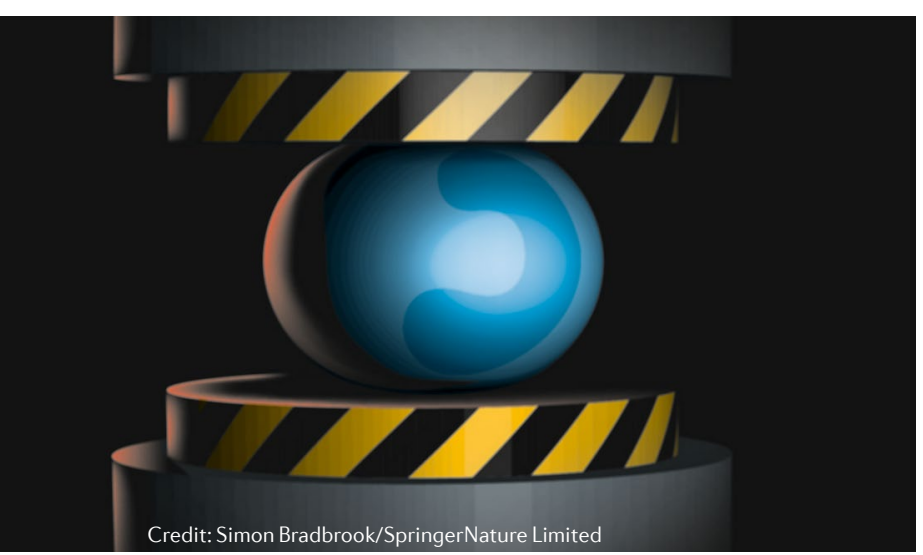

upregulation of pro-inflammatory genes, such as Il1b, CXcl10 and Ptgs2. By contrast, this CHP-induced transcriptional reprogramming did not occur in BMDMs lacking expression of PIEZO1 (derived from Piezo $1^{\mathrm{t} / \mathrm{fl}}$ mice crossed with Piezo1 LysM-Cre mice to generate Piezo ${ }^{\triangle \mathrm{LysM}}$ mice). This observation suggests that myeloid cells, through PIEZO1, can sense pressure alterations in their environment and integrate this with a pro-inflammatory response.

Digging deeper into the RNA sequencing data, the authors noted that many of the upregulated genes were targets of hypoxia-inducible factor (HIF) proteins. Indeed, CHP stimulation led to HIFla protein accumulation and translocation to the nucleus in Piezo $1^{\mathrm{f} / \mathrm{fl}}$ BMDMs but not in Piezo ${ }^{\triangle \mathrm{Lys} \mathrm{M}}$ BMDMs. Moreover, HIF1a-deficient BMDMs failed to show a pro-inflammatory response to CHP. The increase in HIF1a protein was not the result of hypoxic conditions or changing $\mathrm{pH}$ in the pressure chamber nor did it involve gene transcription. Instead, the authors suggest that PIEZO1 signalling results in stabilization of the HIF1 $\alpha$ protein.

To explore the mechanism of HIF1a stabilization, the authors inhibited the PIEZO1-mediated calcium influx into the cytoplasm. BMDMs exposed to calcium-free medium or a PIEZO1 inhibitor failed to accumulate HIF1 $\alpha$ protein, indicating a functional role for the PIEZO1 channel. HIF1a stabilization could also be mediated by a soluble factor present in the supernatant of BMDMs exposed to CHP.

Further re-examination of the RNA sequencing data led the authors to define the CHP-induced soluble factor as the hormone endothelin 1 , which is post-translationally processed and secreted as a biologically active peptide. Blockade of endothelin 1 secretion or presence of an endothelin receptor antagonist completely abrogated HIF1 $\alpha$ stabilization and the transcriptional reprogramming following CHP exposure. Based on further analysis and data from previous studies, the authors propose a pathway in which in response to CHP, PIEZO1dependent calcium influx results in the activation of AP-1, which drives the transcription and release of endothelin 1. In turn, endothelin 1 triggers stabilization of HIFla, which promotes pro-inflammatory cytokine expression.

Finally, the authors explored the physiological role for PIEZO1dependent mechanosensation. Following intranasal infection with the bacteria Pseudomonas aeruginosa, Piezo $1^{\Delta \mathrm{LysM}}$ mice had higher bacterial loads, reduced pulmonary immune cell recruitment and decreased levels of inflammatory mediators compared with Piezo1 $1^{\mathrm{f} / \mathrm{fl}}$ littermates. The protective effect of PIEZO1 was dependent on monocyte-derived endothelin 1, as it was abrogated by depletion of monocytes or administration of endothelin 1-blocking antibody. Conversely, Piezo $1^{\Delta \mathrm{LysM}}$ mice showed reduced lung damage in a model of pulmonary fibrosis, which suggests that PIEZO1 may drive unwelcome autoinflammation in fibrotic disease. Thus, PIEZO1 signalling in monocytes induced by pressure changes following entry into the lungs leads to endothelin 1 production and HIF1a stabilization, which induces pro-inflammatory gene expression required for neutrophil recruitment and pathogen clearance, but may also drive immune pathology in fibrotic disease.

ORIGINAL ARTICLE Solis, A. G. et al. Mechanosensation of cyclical force by PIEZO1 is essential for innate immunity. Nature https:// doi.org/10.1038/s41586-019-1485-8 (2019) RELATED ARTICLE Walmsley, S. R. Pressure regulates immune-cell function. Nature https:// doi.org/10.1038/d41586-019-02339-4 (2019) 